\title{
Effectiveness of Automatic Correction of Systematic Effects in Measuring Chains
}

\author{
Mykhaylo Dorozhovets \\ Department of Metrology and Diagnostic Systems, Faculty of Electrical and Computer Engineering Rzeszow University of \\ Technology,ul. Wincentego Pola 2A,35-959,Rzeszów,Poland,michdor@prz.edu.pl
}

\begin{abstract}
The uncertainty of measurements associated with the following correction methods: advanced correction of additive linear drift, correction of additive and multiplicative effects, as well as joint correction of a linear drift and systematic additive and multiplicative effects is analyzed in the present article. For each correction method sensitivity coefficients and amplitude responses according to which noise and internal and external interferences influence the corrected measurement result have been determined. Besides uncertainty of reference quantities, the main factors which limit the efficiency of correction are: non-linearity of measurement function including non-linearity of ADC, no idealities of the switching systems and external and internal noises and periodic interferences. The efficiency of correction of systematic additive and multiplicative effects was studied for the multifunction 16 bit PCI DAQ of family NI 6250.
\end{abstract}

Keywords: Systematic effects, drift, correction, noise, uncertainty.

\section{INTRODUCTION}

It is generally accepted that in order to ensure the required accuracy of measurement result the systematic effects (which are presented in measurement) should be corrected. Namely, according to GUM [1] "It is assumed that the result of a measurement has been corrected for all recognized significant systematic effects and that every effort has been made to identify such effects". On the other hand, it is clear that the correction of systematic effects cannot be carried out in an ideal way as also recognized in the GUM [1]: "The result of a measurement after correction for recognized systematic effects is still only an estimate of the value of the measurand because of the uncertainty arising from random effects and from imperfect correction of the result for systematic effects". This statement mentions two main reasons for limiting the effectiveness of the correction: the impact of random effects and the uncertainty of corrections. In [3] from an analysis of the correction of systematic effects concludes that: "the true value of a correction should be considered as imprecisely evaluable as the true value of any 'input quantity', and of the measurand itself" and "distinction between 'input quantities' and 'corrections' is not justified and not useful". Since each correction is carried out with uncertainty, the effect of the uncertainty of the correction must be considered in the same way as the other components of the measurement uncertainty [2], [3], [4]. One of the possible ways to comply with the GUM requirements, i.e. to solve the issue of the correction of systematic effects, including the assessment of the uncertainty when the result of correction is determined, is to treat all systematic and other influenced quantities and appropriate corrections equally with the input quantities in the functional dependence of the measurand, as in the functional relationship for indirect measurement [2], [3], [4]. This approach (including all corrections in the measurement equation) has been implemented in the present article in relation to the analysis of the quality of automatic correction of systematic effects in industrial measurements. The operating conditions of such measurements are characterized by a large range of influence quantities changes, power supply instability, and intense noise and interference. Sometimes it may turn out that the correction of a systematic effect may cause uncertainty greater than uncertainty when correction is not used. Then the question arises: "Why and when corrections may not be applied" [3]. The answer to this question requires a detailed analysis of all aspects related to each systematic effect and to all other internal and external effects that influence the corrected result. The aim of this article is to investigate the frequently used correction methods from the point of view of detailed analysis of the influence on the corrected result of the factors related to the measuring instrument itself, such as: quantization effects in ADC, non-linearity of functions, parameters of switching systems, as well as uncertainty of used reference quantities and the influence of random noise and periodic interferences. 


\section{BRIEF DESCRIPTION OF THE ANALYZED SYSTEMATIC INFLUENCES AND METHODS OF THEIR AUTOMATIC CORRECTION}

The problem of correction of systematic influences is essentially complicated in industrial measurements. Because such measurements significantly differ from the measurements in specialized metrology laboratories, corrections of the systematic effects should often be carried out without the direct participation of the operator, i.e. automatically and, therefore, without a detailed analysis of the specific impact values. In recent decades, the industrial measurements are more often based on the uses of a computer-based measuring card (so called DAQ - Data Acquisition module). The main parts of a measuring chain based on a typical (universal) DAQ are the input signal conditioners, for example analog multiplexer, input amplifier, filter, etc. and analog-to-digital converter (ADC) and usually a computation component. Measurement result is evaluated using the appropriate program in which the signal processing algorithm is realized. The influencing quantities, such as: temperature, humidity, magnetic, electrostatic, electromagnetic fields, etc., parasitic (capacitance, inductance, resistance, ground loops) connections around the apparatus, machines and electrical power systems, etc. cause the changes of the parameters of the measuring chain components. Under industrial measurement conditions, these effects are not stable over time and it is therefore practically impossible to estimate their exact values. Also, noises and interferences are not stable. In such cases the uncertainty of measurement with corrections is usually determined a priori, during the stage of development of the correction method, using maximum permissible values of influence quantities and expected noise and interference levels. The values of the sensitivity coefficients, which are dependent on the properties of the correction method, should also be determined in advance. Under operating conditions, an effective correction is only possible if all the significant sources are taken into account and uncertainty caused by them is determined adequately [5], [6].

The implementation of each correction requires the prior precise definition of the three main components of the measurement process [7]:

- the measurand,

- the measuring instrument (system),

- the conditions under which the measurement is carried out.

In subsequent studies it is assumed that the measurand should be constant during all measurements, which are made for correction.

Generally the main effects in the measuring chain, which may cause additional components of measurement uncertainty, can be divided into two categories:

(i) those, which will be corrected:

- systematic (approximately constant in time): additive

$\Delta_{0}$, so-called "offset error" independent on quantity $x$ to be measured (measurand) and multiplicative $\delta_{\mathrm{m}} \cdot x$, so-called "gain error", which is proportional to the value of measurand $x$;

- time drifts $d r(t)$ [8], [9], which values are changed in time with slow (comparatively to the time of measurement) speed $s_{\mathrm{dr}}$, due to this time drifts often can be approximated by linear function of time: $d r(t)=s_{\mathrm{dr}} \cdot\left(t-t_{0}\right)$, where $t_{0}$ is a start of the measurement:

$$
\Delta(t)=\Delta_{0}+\delta_{\mathrm{m}} x+s_{\mathrm{dr}}\left(t-t_{0}\right)
$$

(ii) those, which are not corrected, however, significantly affecting the effectiveness of the correction, main examples are:

- random noises $\eta(t)$ and periodical interferences $\varsigma(t)$ of frequency $f$;

- non-ideality of the actual function $F_{\mathrm{r}}(x)$ of the measuring chain, mainly related to non-linearity.

Therefore, in some simplification, the measuring chain (system) can be described by an equation in which the output $Y$ (observed quantity) depends on the measurand $x$, the abovementioned components (1), the values of which depend on the influence quantities, and the noise and periodic interferences:

$$
Y=F_{\mathrm{r}}\left\lfloor x+\Delta_{0}+\delta_{\mathrm{m}} x+s_{\mathrm{dr}}\left(t-t_{0}\right)+A_{\eta}(\eta(t))+A_{\varsigma}(\varsigma(t))\right\rfloor,
$$

where $A_{\eta}(\cdot)$ and $A_{\zeta}(\cdot)$ are the operators that convert noise and interferences into changes in measured values.

In practice, correction of the abovementioned systematic effects generally is based on additional measurements (besides the measurement of measurand) and in general can be achieved in two ways: (i) by measurement of influenced quantities which, taking into account the sensitivity, are used to correct the measurement result and (ii) by using additional measurements (made by the same instrument, the systematic effects of which should be corrected) of certain functions of the measurand and/or reference quantities $X_{\text {ref }}$ and subsequent calculation of the corrected result [10], [11]. In this article we will not analyze the first method and will focus on the second method as the basis for automatic correction.

Most often depending on the level of the components of systematic influences, in the methods, based on additional measurements, are corrected: (i) only additive effects $\left(\Delta_{0}\right)$, (ii) only multiplicative effects $\left(\delta_{\mathrm{m}}\right)$, (iii) combined additive effects and time drifts $\Delta_{0}+s_{\mathrm{dr}}\left(t-t_{0}\right)$, or (iv) additive and multiplicative effects $\Delta_{0}+\delta_{\mathrm{m}} x$.

Advanced correction of additive effect and time drifts. Correction of the systematic additive effects and time drifts may be implemented without reference quantities, using some form of chopping or modulation of input signal [8], 
[9]. In [9] the advanced method of such correction is proposed. In this method four measurements are carried out in equidistance time interval $T$ in the time moments: $t_{1}$ measurement of $+x, t_{2}=t_{1}+T$ - measurement of inverse $-x$, $t_{3}=t_{1}+2 T-$ measurement of inverse $-x, t_{4}=t_{1}+3 T-$ measurement of $+x$. The mathematical models of sequenced indications of measuring instrument and model of corrected result of this method correction are shown in the first line of Table 1.

The systematic multiplicative effects can be corrected, for example, by applying the DEM (dynamic element matching) technology to voltage amplifiers and dividers [9], [12]. However, for amplifiers and dividers, etc., used in measuring modules, this technology cannot be applied and, therefore, it is not analyzed in this paper.
Joint correction of additive and multiplicative effects. The standard method of combined correction of additive and multiplicative effects is based on three measurements [8], [9]: zero input value $(x=0)$, measurand $x$, and reference quantity $X_{\text {ref. In this method there are six possible sequences }}$ of measurement in total. But taking into account the time inverse symmetry only three different sequences are possible (Table 2,a). As will be shown below, the sequence of measurements has a significant impact on the quality of the corrected result. The cause of this is the influence of periodic interferences during the subsequent measurements. The mathematical models of sequenced indications for the first sequence in Table 2,a and model of corrected result, which is independent of the sequence of measurements, are shown in the second line of Table 1.

Table 1. The mathematical models of sequenced measurements and corrected results.

\begin{tabular}{|c|c|c|c|}
\hline & $\begin{array}{l}\text { Corrected } \\
\text { influences }\end{array}$ & $\begin{array}{l}\text { Sequences and mathematical models of } \\
\text { measurements }\end{array}$ & Corrected result \\
\hline 1 & $\begin{array}{l}\text { Additive }+ \\
+ \text { time drift }\end{array}$ & $\begin{array}{l}\text { 1) }+x: N_{\mathrm{x} 1}=x+\Delta_{0}+s_{\mathrm{dr}} \cdot 0 T \\
\text { 2) }-x: N_{\mathrm{x} 2}=-x+\Delta_{0}+s_{\mathrm{dr}} \cdot 1 T \\
\text { 3) }-x: N_{\mathrm{x} 3}=-x+\Delta_{0}+s_{\mathrm{dr}} \cdot 2 T \\
\text { 4) }+x: N_{\mathrm{x} 4}=x+\Delta_{0}+s_{\mathrm{dr}} \cdot 3 T\end{array}$ & $x_{\mathrm{cor}, 3}=\frac{N_{\mathrm{x} 1}-N_{\mathrm{x} 2}-N_{\mathrm{x} 3}+N_{\mathrm{x} 4}}{4} \equiv x$ \\
\hline 2 & $\begin{aligned} & \text { Additive }+ \\
+ & \text { multiplicative }\end{aligned}$ & $\begin{array}{l}\text { 1) } 0: N_{0}=0+\Delta_{0}+\delta_{\mathrm{m}} 0 \\
\text { 2) } x: N_{\mathrm{x}}=x+\Delta_{0}+\delta_{\mathrm{m}} x \\
\text { 3) } X_{\mathrm{ref}:} N_{\text {ref }}=X_{\text {ref }}+\Delta_{0}+\delta_{\mathrm{m}} X_{\text {ref }}\end{array}$ & $x_{\mathrm{cor}, 4}=\frac{N_{\mathrm{x}}-N_{0}}{N_{\mathrm{ref}}-N_{0}} X_{\mathrm{ref}} \equiv x$ \\
\hline 3 & $\begin{array}{c}\text { Additive }+ \\
+ \text { multiplicative }+ \\
+ \text { time drift, I }\end{array}$ & $\begin{array}{l}\text { 1) }+x: N_{x 1}=x \cdot\left(1+\delta_{m}\right)+\Delta_{0}+s_{\mathrm{dr}} \cdot 0 T \\
\text { 2) }-X_{\text {ref: }} N_{\text {ref1 }}=-X_{\text {ref }} \cdot\left(1+\delta_{\mathrm{m}}\right)+\Delta_{0}+s_{\mathrm{dr}} \cdot 1 T \\
\text { 3) }-x: N_{\mathrm{x} 2}=-x \cdot\left(1+\delta_{\mathrm{m}}\right)+\Delta_{0}+s_{\mathrm{dr}} \cdot 2 T \\
\text { 4) }+X_{\text {ref: }} N_{\text {ref2 }}=X_{\text {ref }} \cdot\left(1+\delta_{\mathrm{m}}\right)+\Delta_{0}+s_{\mathrm{dr}} \cdot 3 T\end{array}$ & $x_{\mathrm{cor}, 5}=\frac{N_{\mathrm{ref} 1}+N_{\mathrm{ref} 2}-2 N_{\mathrm{x} 2}}{N_{\mathrm{x} 1}+N_{\mathrm{x} 2}-2 N_{\mathrm{ref} 1}} \cdot X_{\mathrm{ref}} \equiv x$ \\
\hline 4 & $\begin{array}{c}\text { Additive }+ \\
+ \text { multiplicative }+ \\
+ \text { time drift, II }\end{array}$ & $\begin{array}{l}\text { 1) }+x: N_{x 1}=x \cdot\left(1+\delta_{\mathrm{m}}\right)+\Delta_{0}+s_{\mathrm{dr}} \cdot 0 T \\
\text { 2) - } x: N_{\mathrm{x} 2}=-x \cdot\left(1+\delta_{\mathrm{m}}\right)+\Delta_{0}+s_{\mathrm{dr}} \cdot 1 T \\
\text { 3) - } X_{\text {ref: }} N_{\text {ref } 1}=-X_{\text {ref }} \cdot\left(1+\delta_{\mathrm{m}}\right)+\Delta_{0}+s_{\mathrm{dr}} \cdot 2 T \\
\text { 4) }+X_{\text {ref: }} N_{\text {ref } 2}=X_{\text {ref }} \cdot\left(1+\delta_{\mathrm{m}}\right)+\Delta_{0}+s_{\mathrm{dr}} \cdot 3 T\end{array}$ & $x_{\mathrm{cor}, 6}=\frac{3 N_{\mathrm{x} 1}-5 N_{\mathrm{x} 2}+N_{\mathrm{ref} 1}+N_{\mathrm{ref} 2}}{3 N_{\mathrm{ref} 2}-5 N_{\mathrm{ref} 1}+N_{x 1}+N_{\mathrm{x} 2}} \cdot X_{\mathrm{ref}} \equiv x$ \\
\hline
\end{tabular}

Joint correction of additive and multiplicative effects and linear time drift. For a joint correction of linear drift and systematic additive and multiplicative effects, a method based on a combination of the methods described by (3) and (4) can be used. However, using methods (3) and (4) directly, eight measurements are needed to obtain the corrected result. Because in each measurement indication the additive $\Delta_{0}$, multiplicative $\delta_{\mathrm{m}} x$, and linear time drift $s_{\mathrm{dr}} \cdot\left(t-t_{0}\right)$ are described by three parameters, therefore, combination of these effects can be corrected by a minimum of four measurements, which are carried out with the same time delay $T$. On the basis of the advanced method (3) for the correction of linear time drift in the two central measurements, the inverse values of the measured and reference quantity should be calculated. Taking into account the time inverse symmetry of the sequence of measurements, only two different sequences are possible (Table 2,b).

Table 2. The sequence of measurements for the correction of additive and multiplicative effects (a), and in a joint correction of additive and multiplicative effects and linear time drift (b).

\begin{tabular}{|c|c|c|c|c|c|c|c|c|}
\hline a & \multicolumn{3}{|c|}{ Measured quantity } & $\mathrm{b}$ & \multicolumn{4}{|c|}{ Measured quantity } \\
\hline 1 & 0 & $x$ & $X_{\text {ref }}$ & 1 & $x$ & $-X_{\text {ref }}$ & $-x$ & $X_{\text {ref }}$ \\
\hline 2 & 0 & $X_{\text {ref }}$ & $x$ & 2 & $x$ & $-x$ & $-X_{\text {ref }}$ & $X_{\text {ref }}$ \\
\hline 3 & $x$ & 0 & $X_{\text {ref }}$ & \multicolumn{4}{|c|}{} \\
\hline
\end{tabular}




\section{ANALYSIS OF THE IMPACT OF THE MAIN FACTORS WHICH LIMIT THE EFFECTIVENESS OF CORRECTION}

The effectiveness of correction of systematic additive and multiplicative and linear time drift is limited by many reasons. The main sources, which decrease effectiveness of automatic corrections, are:

- uncertainty of the reference quantities, which are used in correction;

- effect of quantization of measurement results in ADC;

- non-linearity of function of the measuring chain;

- switching effects;

- random noises and periodic interferences and other variations of repeated observations, etc.

It is clear that if in correction a reference quantity $X_{\text {ref }}$ is used, then even in the absence of the abovementioned other factors, the uncertainty of the corrected result is limited by the uncertainty $u_{\mathrm{B}}\left(X_{\text {ref }}\right)$ of the reference quantity. Assuming rectangular distribution of possible values within $\pm \delta_{\text {ref }}\left|X_{\text {ref }}\right|$ (where $\delta_{\text {ref }}$ is a relative maximal permissible error (MPE) of $X_{\text {ref }}$ ), the component of a combined standard uncertainty is given by expression:

$$
u_{\mathrm{B}}(x)_{\lim }=\left|C_{\mathrm{ref}}\right| \cdot u_{\mathrm{B}}\left(X_{\mathrm{ref}}\right)=\frac{\left|C_{\mathrm{ref}}\right| \cdot \delta_{\mathrm{ref}} X_{\mathrm{ref}}}{\sqrt{3}}
$$

where $C_{\text {ref }}$ is a sensitivity coefficient for a reference quantity. The value $u_{\mathrm{B}}(x)_{\lim }$ determines the theoretical effectiveness of the correction.

Quantization, resolution of $A D C$. The resolution of used $\mathrm{ADC}$ or the number of significant digits of used digital meter is very important in the quality of the corrected result. The component of combined standard uncertainty of a corrected result caused by quantization effect in ADC is given by a known formula:

$$
u_{\mathrm{B}}(x)_{\mathrm{q}}=\left|C_{\mathrm{q}}\right| \cdot \frac{\mathrm{LSD}}{2 \sqrt{3}}=\left|C_{\mathrm{q}}\right| \cdot \frac{X_{\mathrm{R}}}{2 \sqrt{3} \cdot 2^{n}},
$$

where $C_{\mathrm{q}}$ is a sensitivity coefficient for quantization in $\mathrm{ADC} ; X_{\mathrm{R}}$ is range; $n$ is number of bits $\mathrm{ADC}$, $\mathrm{LSD}=X_{\mathrm{R}} / 2^{n}=q$ is a quantum - a value of the least significant bit (or digit) of indication.

Non-linearity. Two components of non-linearity, differential and integral, are traditionally considered separately. The effect of differential non-linearity of ADC is associated with a change in the values of adjacent quantums, slightly different from LSB. In the data sheet of ADC or measuring DAQ card we can find the limit of differential non-linearity in units of the least significant bit: $\Delta_{\mathrm{nl}, \mathrm{dif}}= \pm \alpha_{\mathrm{nl}, \mathrm{dif}} \cdot q$ (or bit), where usually $\alpha_{\mathrm{nl}, \mathrm{dif}}<1$. Therefore, the effect of differential non-linearity onto uncertainty of the corrected result is similar to the effect of quantization, i.e. it can be estimated by an expression similar to (8):

$$
u_{\mathrm{B}}(x)_{\mathrm{nl}, \mathrm{dif}}=\alpha_{\mathrm{nl}, \mathrm{dif}} u_{\mathrm{B}}(x)_{\mathrm{q}} .
$$

Integral non-linearity (Fig. 1) in data sheet usually is declared as normalized to the range $X_{\mathrm{R}}$ maximal deviation $\Delta_{\mathrm{nl}, \text { lim }}$ from the straight line: $\gamma_{\mathrm{nl}, \text { int }}= \pm \Delta_{\mathrm{nl}, \text { lim }} / X_{\mathrm{R}}, \%$. Thus, assuming rectangular probability of possible values of $\Delta_{\mathrm{nl} \text {,int }}$ the component of combined standard uncertainty associated with integral non-linearity can be calculated as:

$$
u_{\mathrm{B}}(x)_{\mathrm{nl}, \mathrm{int}}=\left|C_{\mathrm{nl}}\right| \cdot \frac{\gamma_{\mathrm{nl}, \mathrm{int}} \cdot X_{\mathrm{R}}}{\sqrt{3}},
$$

where $C_{\mathrm{nl}}$ is a sensitivity coefficient for integral nonlinearity.

If non-linearity of the function of measuring chain is monotonic and in a first approximation can be described by a parabolic function $\Delta_{\mathrm{nl} \text {,int }}(x)=\varepsilon_{\mathrm{nl}} \cdot x^{2}$ (Fig. 1), then using the value of reference quantity $X_{\text {ref }}=2 \cdot(\sqrt{2}-1) X_{\mathrm{R}} \approx 0.828 \cdot X_{\mathrm{R}}$ after correction of gain systematic component, the maximal impact of non-linearity onto the corrected result will be minimal:

$$
\Delta_{\mathrm{nl}, \mathrm{o}, \max }=\varepsilon_{\mathrm{nl}} \cdot X_{\mathrm{R}}^{2} \cdot(3-2 \sqrt{2}) \approx 0.686 \cdot \gamma_{\mathrm{nl}, \mathrm{int}} \cdot X_{\mathrm{R}} .
$$

I.e., using a value of reference quantity $X_{\text {ref }} \approx 0.828 \cdot X_{\mathrm{R}}$, instead of, for example $X_{\text {ref }}=X_{\mathrm{R}}$, the maximal integral non-linearity will decrease by approximately $30 \%$.

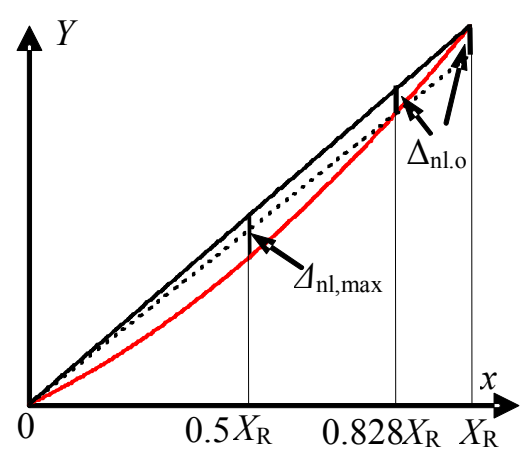

Fig. 1. Optimal correction of gain error when integral non-linearity is parabolic.

Switches. In all the above described correction methods, connection and disconnection or/and inverting of the quantity to be measured and/or the reference quantity shall be realized. For this purpose the switching systems are used. Real switches are not ideal, there are many parameters of switches which affect the input and reference signals. The main representatives are: contact potential, offset current, contact resistance, leakage currents, ground loops, interferences, etc. [13]. Precision measurements usually use differential connection of signal sources, so in each channel a pair of the same switches is used. However, the parameters 
of both switches are never the same, they are characterized by a certain dispersion of appropriate values of their parameters [13]. In many cases, when low-voltage must be connected / disconnected, the most negative affect provides the contact thermoelectric offset voltage of switch elements [13]. Even if a matching contact pair is used, the total cancellation of contact potential cannot be achieved because temperature differences in both switches will cause slightly different contact potentials [13]. The uncompensated difference $\Delta e_{\mathrm{sw}}$ in the contact potentials of such switch pairs can reach \pm several microvolts. When input quantity is voltage assuming rectangular distribution on a contact potential, the component of a combined standard uncertainty can be calculated by the formula:

$$
u_{\mathrm{B}}(x)_{\mathrm{sw}}=\left|C_{\mathrm{sw}}\right| \cdot \frac{\Delta e_{\mathrm{sw}}}{\sqrt{3}}
$$

where $C_{\mathrm{sw}}$ is a sensitivity coefficient for a contact potential.

Since each correction needs several measurements, i.e. switching of measured and reference quantities, the setting times of the switches should be taken into account.

In each specific case, depending on the quantity to be measured, the used method of correction and the type of used switching system, the negative impact of the switching should be analyzed individually.

Because the effects of quantization and differential and integral non-linearity are related to the same result obtained in $\mathrm{ADC}$, the relevant sensitivity coefficients is the same:

$$
C_{\mathrm{q}}=C_{\mathrm{dnl}}=C_{\mathrm{nl}}=C_{\mathrm{ADC}} \cdot
$$

Noises and periodic interferences. Present in all measurements, noises and interferences can be classified into two categories:

1) external, which come with input signal (normal and common mode) and

2) internal, caused by internal sources in the components of measuring chain, such as thermal (Johnson) and another kind of noises, and also power supply, digital clock and other digital elements inside of the measuring instrument.

A difference between the effects of internal and external interferences and noises must be taken into account. Random noises (external $\eta_{\text {ext }}(t)$ and internal $\left.\eta_{\text {int }}(t)\right)$ usually are described by spectral densities $N_{\text {ext }}(f), N_{\text {int }}(f)$, or standard deviation $\left(V_{\mathrm{RMS}}\right)$, or peak-to-peak value $\left(V_{\mathrm{p}-\mathrm{p}}\right)$.

The most common sources of periodical interferences (external $\varsigma_{\text {ext }}(t)$ and internal $\varsigma_{\text {int }}(t)$ ) are power supply systems (typically of 50 or $60 \mathrm{~Hz}$ with higher harmonics), operation of radio, telecommunications and other such systems, electrical machinery and transport, electronic apparatus, digital electronic devices, etc. Such interferences are usually described by the maximal values of amplitude

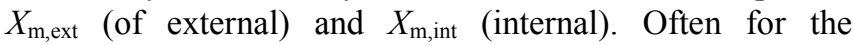
components of measuring chain are declared the NMRR (normal mode rejection ratio) and CMRR (common mode rejection ratio), which values also determine the impact of these interferences onto corrected result [8]. These noises and interferences cause the variance $\sigma_{\eta+\varsigma}^{2}$ in repeated observations and in assumption that these sources are independent it can be calculated as a sum of components:

$$
\begin{aligned}
& u_{\mathrm{c}}^{2}(x)_{\eta+\varsigma}=\sigma_{\eta+\varsigma}^{2}= \\
& =C_{\eta, \mathrm{ext}}^{2} \cdot \sigma_{\eta, \mathrm{ext}}^{2}+C_{\eta, \text { int }}^{2} \cdot \sigma_{\eta, \text { int }}^{2}+C_{\varsigma, \text { ext }}^{2} \cdot \sigma_{\varsigma, \mathrm{ext}}^{2}+C_{\varsigma, \text { int }}^{2} \cdot \sigma_{\varsigma, \text { int }}^{2}
\end{aligned}
$$

where $\sigma_{\eta, \text { ext }}^{2}, \sigma_{\eta, \text { int }}^{2}, \sigma_{\varsigma, \text { ext }}^{2}, \sigma_{\varsigma, \text { int }}^{2}$ are the variations of the external and internal random noises and periodic interferences, respectively; $C_{\eta, \text { ext }}, C_{\eta, \text { int }}, C_{\varsigma, \text { ext }}, C_{\varsigma, \text { int }}$ are the sensitivity coefficients for these influences.

Thus, the variances caused by the external and internal noises are given by the well-known formulae:

$$
\begin{gathered}
\sigma_{\eta, \mathrm{ext}}^{2}=2 \int_{0}^{\infty} A_{\mathrm{sp}}^{2}(f) \cdot A_{\mathrm{ext}}^{2}(f) \cdot N_{\mathrm{ext}}(f) d f, \\
\sigma_{\eta, \mathrm{in} t}^{2}=2 \int_{0}^{\infty} A_{\mathrm{sp}}^{2}(f) \cdot A_{\mathrm{int}}^{2}(f) \cdot N_{\mathrm{int}}(f) d f,
\end{gathered}
$$

where $A_{\text {sp }}(f)$ is an amplitude response (AR) of the signal processing algorithm (included conversion in ADC) realized in measuring chain, $A_{\text {ext }}(f)$ and $A_{\text {int }}(f)$ are the appropriate amplitude responses (in relation to the external and internal effects), which depend on the used correction algorithm.

Similarly, assuming arcsine probability density distribution of interferences of amplitudes $X_{\mathrm{m} \text {,ext }}$ and $X_{\mathrm{m} \text {,int }}$ and frequency $f$, the standard deviations are given by the formulae:

$$
\sigma_{\zeta, \text { ext }}=\frac{X_{\mathrm{m}, \mathrm{ext}}}{\sqrt{2}} A_{\mathrm{sp}}(f) A_{\mathrm{ext}}(f), \sigma_{\zeta, \mathrm{int}}=\frac{X_{\mathrm{m}, \text { int }}}{\sqrt{2}} A_{\mathrm{sp}}(f) A_{\mathrm{int}}(f) .
$$

\section{ANALYSIS OF THE AMPLITUDE RESPONSES}

As mentioned above it is necessary to determinate the $A_{\text {ext }}(f)$ and $A_{\text {int }}(f)$ separately. For this purpose we will use the complex exponential function $X_{\mathrm{m}} e^{j \omega \cdot t_{i}}$, where $\omega=2 \pi f, f$ is a frequency and $X_{\mathrm{m}}$ is an amplitude of influenced component, $t_{i}$ are the appropriated times of the consequent measurements. For all correction methods time interval between adjacent measurements is constant and equal $T$, zero of time axis is located in the midpoint between the first and last measurements. Therefore, for the methods (3), (5) and (6): $t_{1}=-3 T / 2, t_{2}=-T / 2 ; t_{3}=T / 2, t_{4}=3 T / 2$. In method (4): $t_{1}=-T$ (meas. 0), $t_{2}=0$ (meas. $x$ ) and $t_{3}=T$ (meas. $X_{\text {ref }}$ ).

The external and internal influences cause the change of indications of measuring instrument and as a result the change of corrected result $x_{\text {cor }}(j f)$. Then the corresponding $A_{\text {ext }}(f)$ and $A_{\text {int }}(f)$ are given by the formulae: 


$$
A_{\text {ext }}(f)=\frac{\left|x_{\text {cor,ext }}(j f)-x\right|}{X_{\mathrm{m}}}, A_{\text {int }}(f)=\frac{\left|x_{\text {cor,int }}(j f)-x\right|}{X_{\mathrm{m}}}
$$

In method (3) during four measurements the external influences cause the following changes of indications: $N_{x 1}=x+X_{m} \cdot e^{j 3 \pi f T}, \quad N_{x 2}=-x-X_{\mathrm{m}} \cdot e^{j \pi f T}$, $N_{x 3}=-x-X_{\mathrm{m}} \cdot e^{-j \pi f T}$ and $N_{x 4}=x+X_{\mathrm{m}} \cdot e^{-j 3 \pi f T}$. Similarly, the internal influences cause the following changes of indications: $\quad N_{x 1}=x+X_{\mathrm{m}} \cdot e^{j 3 \pi f T}, \quad N_{x 2}=-x+X_{\mathrm{m}} \cdot e^{j \pi f T}$, $N_{x 3}=-x+X_{\mathrm{m}} \cdot e^{-j \pi f T}$ and $N_{x 4}=x+X_{\mathrm{m}} \cdot e^{-j 3 \pi f T}$. For these indications corrected results are:

$$
\begin{aligned}
& x_{\mathrm{cor}, \mathrm{ext}, 3}(f)=x+X_{\mathrm{m}} \frac{e^{j 3 \pi f T}+e^{j \pi f T}+e^{-j \pi f T}+e^{-j 3 \pi f T}}{4}, \\
& x_{\mathrm{cor}, \mathrm{int}, 3}(f)=x+X_{\mathrm{m}} \frac{e^{j 3 \pi f T}-e^{j \pi f T}-e^{-j \pi f T}+e^{-j 3 \pi f T}}{4}
\end{aligned}
$$

Using (18) in (17), the appropriate AR are presented in the first row of Table 3 and are shown in Fig. 2,a, and Fig. 2,b.

From Fig. 2,a, Fig. 2,b we can see that if the basis frequency of periodical internal and external interference is $f$, then for the measurement interval $T=1 / 2 f$ exactly, these interferences will be rejected. If the interference frequency $f$ deviates from the nominal value $f_{\text {nom, }}$, the influence of such interference depends on the corresponding value $A_{\mathrm{ext}, 3}(f)$ and $A_{\text {int, } 3}(f)$ (Table 3 ).

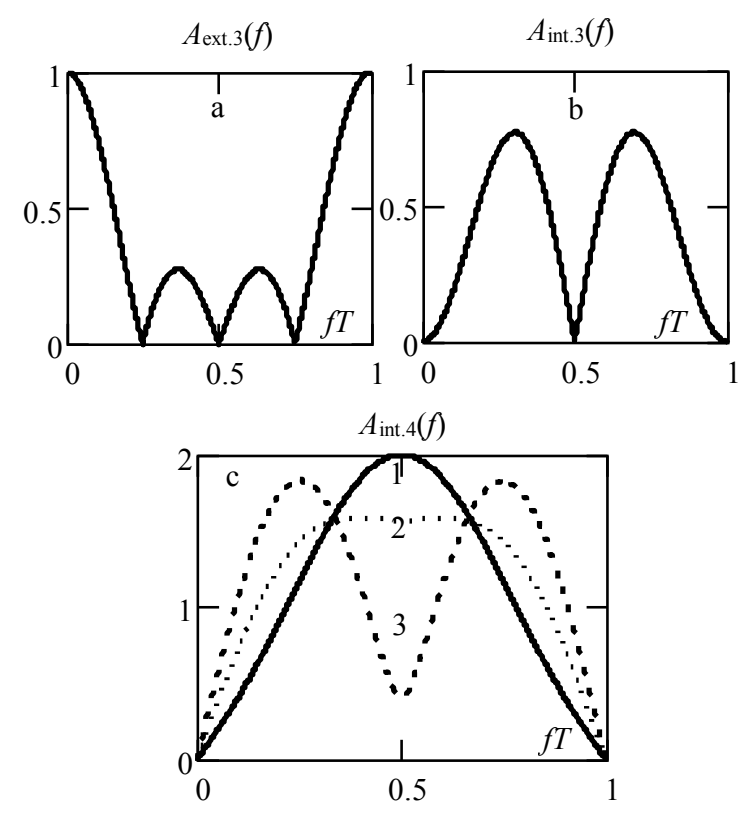

Fig. 2. The amplitude responses related to the external (a) and internal (b) components - method (3) and amplitude responses for the internal components (c) - method (4), 1,2,3 - sequences in Table 2 , a, $x / X_{\text {ref }} \approx 0.8$.
In method (4), because external noises and periodic interferences affected only the measurement of measurand, therefore AR $A_{\text {ext, } 4}(f)=1$. The internal influences cause the following changes of indications: $N_{0}=X_{\mathrm{m}} \cdot e^{j 2 \pi f T}$, $N_{x}=x+X_{\mathrm{m}} \cdot e^{j 0 \pi f T}, N_{\text {ref }}=X_{\text {ref }}+X_{\mathrm{m}} \cdot e^{-j 2 \pi f T}$. Using these values in (4) the corrected result is given by formula:

$$
x_{\mathrm{cor}, \mathrm{in}, 4}(j f)=\frac{x+X_{\mathrm{m}}\left(1-e^{j 2 \pi f T}\right)}{1+\frac{X_{\mathrm{m}}}{X_{\mathrm{ref}}}\left(e^{-j 2 \pi f T}-e^{j 2 \pi f T}\right)} .
$$

From (19) we can see that internal influences non-linearly affect the value $x_{\text {cor,int, } 4}(j f)$ and, therefore, the characteristic $A_{\text {int, } 4}(f)$ is also non-linear. However, internal interferences always meet the condition $X_{\mathrm{m}}<<X_{\text {ref, therefore, the good }}$ approximation of $A_{\text {int, }}(f)$ is presented in the second row of Table 3 and shown in Fig. 2,c.

In correction methods (5) and (6) the external noise and periodic interference influenced the quantity to be measured, so they are included only in the values of $N_{x 1}$ and $N_{x 2}$ in both correction methods. Therefore, for the method (5) - first line in Table 2,b the indications are: $N_{x 1}=x+X_{\mathrm{m}} \cdot e^{j 3 \pi f T}$, $N_{x 2}=-x-X_{\mathrm{m}} \cdot e^{-j \pi f T}$. For the method (6) (second line in Table 2,b the indications are: $N_{x 1}=x+X_{\mathrm{m}} \cdot e^{j 3 \pi f T}$, $N_{x 2}=-x-X_{\mathrm{m}} \cdot e^{j \pi f T}$. Using these values in (5) and (6) the corrected results are given by formulae:

$$
\begin{gathered}
x_{\text {cor.ext, },(j f)}=\frac{x+X_{\mathrm{m}} \cdot e^{-j \pi f T}}{1+\frac{X_{\mathrm{m}}}{X_{\mathrm{ref}}} \cdot \frac{e^{j 3 \pi f T}-e^{-j \pi f T}}{2}}, \\
x_{\text {cor.ext, } 6}(j f)=\frac{x+\frac{X_{\mathrm{m}}}{8} \cdot\left(3 e^{j 3 \pi f T}+5 e^{j \pi f T}\right)}{1+\frac{X_{\mathrm{m}}}{8 X_{\mathrm{ref}}}\left(e^{j 3 \pi f T}-e^{j \pi f T}\right)} .
\end{gathered}
$$

In these cases, because the $X_{\mathrm{m}}<<X_{\text {ref, linearization in (20) }}$ may also be applied. Therefore, after substituting (20) into (17), we can calculate the corresponding responses $A_{\text {ext, } 5}(f)$, $A_{\text {ext, } 6}(f)$, which are given in the third and fourth rows of Table 3 and in Fig. 3,a, Fig. 3,c.

In correction methods (5) and (6) the internal noise and periodic interference influenced all measurements. Accordingly, for the first line of Table 2,b (method (5)) the values of corresponding indications are: $N_{x 1}=x+X_{\mathrm{m}} \cdot e^{j 3 \pi f T}, \quad N_{x 2}=-x+X_{\mathrm{m}} \cdot e^{-j \pi f T} ;$ $N_{\text {ref } 1}=-X_{\text {ref }}+X_{\mathrm{m}} \cdot e^{j \pi f T}, \quad N_{\text {ref } 2}=X_{\text {ref }}+X_{\mathrm{m}} \cdot e^{-j 3 \pi f T}$. For the second line of Table 2,b (method (6)) the values of the indications are: $N_{x 1}=x+X_{\mathrm{m}} \cdot e^{j 3 \pi f T}, N_{x 2}=-x+X_{\mathrm{m}} \cdot e^{j \pi f T}$; 
$N_{\text {ref } 1}=-X_{\text {ref }}+X_{\mathrm{m}} \cdot e^{-j \pi f T}, N_{\text {ref } 2}=X_{\text {ref }}+X_{\mathrm{m}} \cdot e^{-j 3 \pi f T}$. After substituting these values into formulas (5), (6) we have:

$$
\begin{gathered}
x_{\mathrm{cor}, \mathrm{int}, 5}(j f)=\frac{x+\frac{X_{\mathrm{m}}}{2}\left(e^{j \pi f T}-2 e^{-j \pi f T}+e^{-j 3 \pi f T}\right)}{1+\frac{X_{\mathrm{m}}}{2 X_{\mathrm{ref}}}\left(e^{j 3 \pi f T}-2 e^{j \pi f T}+e^{-j \pi f T}\right)}, \\
x_{\text {cor.int }, 6}(j f)=\frac{x+\frac{X_{\mathrm{m}}}{8}\left(3 e^{j 3 \pi f T}+e^{-j 3 \pi f T}-5 e^{j \pi f T}+e^{-j \pi f T}\right)}{1+\frac{X_{\mathrm{m}}}{8 X_{\mathrm{ref}}}\left(3 e^{-j 3 \pi f T}+e^{j 3 \pi f T}-5 e^{-j \pi f T}+e^{j \pi f T}\right)} .
\end{gathered}
$$

After linearization $\left(X_{\mathrm{m}}<<X_{\text {ref }}\right)$ and substituting (21) into (17) we can calculate the corresponding responses $A_{\text {int, } 5}(f)$, $A_{\text {int, } 6}(f)$, which are given in the third and fourth rows of Table 3 and in Fig. 3,b, Fig. 3,d.

From third and fourth lines of Table 3 and Fig. 3 we can see that in the correction method (6) the effect of the internal and external noises and interferences is approximately twice less in comparison with the correction method (5).

If the spectral density of random noises is constant $\left(N_{0}\right)$, then the effect of such noises on the corrected result depends on the mean value (in the $1 / \mathrm{T}$ band) of the square of the corresponding AR:

$$
T \int_{0}^{1 / T} A_{\mathrm{ext}}^{2}(f) d f=C_{e x t}^{2}, T \int_{0}^{1 / T} A_{\mathrm{int}}^{2}(f) d f=C_{\mathrm{int}}^{2},
$$

$A_{\text {ext.5}}(f)$

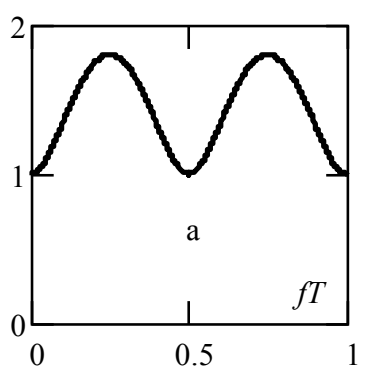

$A_{\text {ext.6}}(f)$

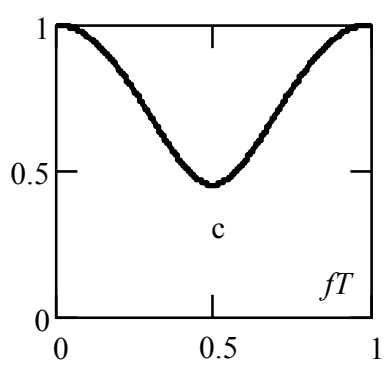

$A_{\text {int. } 5(f)}$

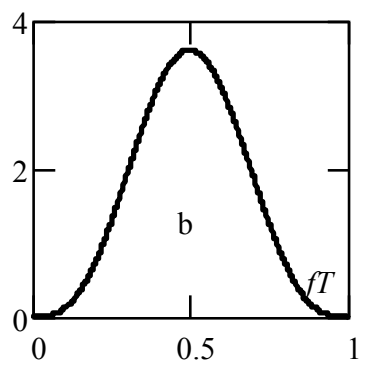

$A$ int.6(f)

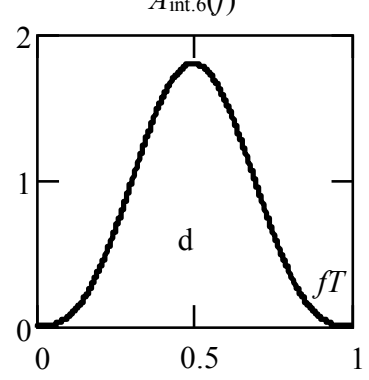

Fig. 3. The amplitude responses related to the external and internal

\begin{tabular}{|c|c|c|}
\hline $\begin{array}{l}\text { Method of } \\
\text { correction }\end{array}$ & External noises and interferences: & Internal noises and interferences: \\
\hline (3) & $\begin{array}{l}A_{\mathrm{ex}, 3}(f)=|\cos (\pi f T) \cdot \cos (2 \pi f T)| \\
T \int_{0}^{1 / T} A_{\mathrm{ext}, 3}^{2}(f) d f=\frac{1}{4}=C_{3, \mathrm{ext}}^{2}=C_{3}^{2}\end{array}$ & $\begin{array}{l}A_{\mathrm{int}, 3}(f)=|\sin (\pi f T) \cdot \sin (2 \pi f T)| \\
T \int_{0}^{1 / T} A_{\mathrm{int}, 3}^{2}(f) d f=\frac{1}{4}=C_{3, \mathrm{int}}^{2}=C_{3}^{2}\end{array}$ \\
\hline (4) & $\begin{array}{l}A_{\mathrm{ext}, 4}(f)=1 \\
C_{4, \mathrm{ext}}^{2}=1\end{array}$ & $\begin{array}{l}A_{\mathrm{int}, 4}(f) \approx 2|\sin (\pi f T)| \cdot \sqrt{1+4 \frac{x_{\mathrm{cor}}}{X_{\text {ref }}}\left(\frac{x_{\mathrm{cor}}}{X_{\text {ref }}}-1\right) \cos ^{2}(\pi f T)} \\
T \int_{0}^{1 / T} A_{\mathrm{int}, 4}^{2}(f) d f \approx 2\left(1-\frac{x_{\mathrm{cor}}}{X_{\text {ref }}}+\left(\frac{x_{\mathrm{cor}}}{X_{\text {ref }}}\right)^{2}\right)=C_{4, \mathrm{int}}^{2}\end{array}$ \\
\hline (5) & $\begin{array}{l}A_{\text {ext }, 5}(f) \approx \sqrt{1+\frac{x_{\text {cor }}}{X_{\text {ref }}}\left(2+\frac{x_{\text {cor }}}{X_{\text {ref }}}\right) \sin ^{2}(2 \pi f T)} \\
T \int_{0}^{1 / T} A_{\text {ext }, 5}^{2}(f) d f \approx 1+\frac{x_{\text {cor }}}{X_{\text {ref }}}+\frac{1}{2}\left(\frac{x_{\text {cor }}}{X_{\text {ref }}}\right)^{2}=C_{5, \text { ext }}^{2}\end{array}$ & $\begin{array}{l}A_{\mathrm{int}, 5}(f) \approx 2 \sin ^{2}(\pi f T) \sqrt{1+\left(\frac{x_{\mathrm{cor}}}{X_{\mathrm{ref}}}\right)^{2}-\frac{2 x_{\mathrm{cor}}}{X_{\mathrm{ref}}} \cos (2 \pi f T)} \\
T \int_{0}^{1 / T} A_{\mathrm{in}, 5}^{2}(f) d f \approx \frac{3}{2}\left(1+\frac{4}{3} \frac{x_{\mathrm{cor}}}{X_{\mathrm{ref}}}+\left(\frac{x_{\mathrm{cor}}}{X_{\text {ref }}}\right)^{2}\right)=C_{5, \text { int }}^{2}\end{array}$ \\
\hline (6) & $\begin{array}{l}A_{\text {ext }, 6}(f) \approx \sqrt{\cos ^{2}(\pi f T)+\frac{1}{16}\left(1+\frac{x_{\text {cor }}}{X_{\text {ref }}}\right)^{2} \sin ^{2}(\pi f T)} \\
T \int_{0}^{1 / T} A_{\text {ext }, 6}^{2}(f) d f \approx \frac{1}{2}\left(1+\frac{1}{16}\left(1+\frac{x_{\text {cor }}}{X_{\text {ref }}}\right)^{2}\right)=C_{6, \text { ext }}^{2}\end{array}$ & $\begin{array}{l}A_{\text {int }, 6}(f) \approx \sin ^{2}(\pi f T) \sqrt{\left[\left(1+\frac{x_{\text {cor }}}{X_{\text {ref }}}\right)^{2} \sin ^{2}(\pi f T)+4\left(1-\frac{x_{\text {cor }}}{X_{\text {ref }}}\right)^{2} \cos ^{2}(\pi f T)\right]} \\
\int_{0}^{1 / T} A_{\text {in }, 6}^{2}(f) d f \approx \frac{9}{16}\left(1+\frac{2}{9} \frac{x_{\text {cor }}}{X_{\text {ref }}}+\left(\frac{x_{\text {cor }}}{X_{\text {ref }}}\right)^{2}\right)=C_{6, \text { int }}^{2}\end{array}$ \\
\hline
\end{tabular}
influences in correction methods (5) and (6), $x / X_{\text {ref }} \approx 0.8$.

Table 3. Amplitude responses for the external and internal noises and interferences. 
The values of the mean squares $A_{\text {ext }}(f)$ and $A_{\text {int }}(f)$ according to (22), which correspond to the analyzed correction methods, are presented in Table 3. In the last three correction methods these values depend on the ratio $x / X_{\text {ref }}$. For simplification purposes in these dependencies the approximation $x=x_{\text {cor }}$ has been applied. This approximation is also used in the next analysis of the combined standard uncertainty of the corrected results.

In all analyzed methods the influences of the internal and external noises and periodic interferences may be reduced by filtering and (or) averaging, so $\left|A_{\text {sp }}(f)\right|<<1$. For example, after digital averaging of $n$ independent values of the random noise, the variance is reduced by $n$ times:

$$
\sigma_{\eta, \mathrm{av}}^{2}=\sigma_{\eta}^{2} / n
$$

However, here we must indicate that increasing the number $(n)$ of averaged random observations by increasing the sample frequency $\left(f_{\mathrm{s}}\right)$ of $\mathrm{ADC}$ may cause autocorrelation of observations and this may essentially decrease the efficiency of averaging [15], [16], [17].

If the uniform averaging of input indications is used and averaging interval $T_{\mathrm{av}}=n / f_{s}$ ( $f_{\mathrm{s}}$ is a sample frequency) is matched to a period $T_{\mathrm{h}}$ of certain harmonic interferences of frequency $f_{\mathrm{h}}: T_{\mathrm{av}}=T_{\mathrm{h}}=1 / f_{\mathrm{h}}$, then rejection of this harmonic can be described by known expression:

$$
\mathrm{NMRR}_{\mathrm{av}}=\frac{X_{\mathrm{m}}}{\left|\Delta X_{\mathrm{m}, \mathrm{av}}\right|_{\max }}=\left|\frac{\sin \left(\pi f_{\mathrm{h}} / f_{\mathrm{s}}\right)}{\frac{1}{n} \sin \left(n \pi f_{\mathrm{h}} / f_{\mathrm{s}}\right)}\right| .
$$

where $\left|\Delta X_{\mathrm{m}, \mathrm{av}}\right|_{\max }$ is a maximal value of the harmonic effect of amplitude $X_{\mathrm{m}}$ after averaging.

It is well-known, when the frequency $f_{\mathrm{h}}$ is unstable and slightly deviated from nominal value $f_{\mathrm{h}, \mathrm{nom}}$ : $\left|\delta_{f}\right|=\left|f_{\mathrm{h}}-f_{\mathrm{h}, \text { nom }}\right| / f_{\mathrm{h}, \text { nom }}<<1$, then this harmonic component will be reduced as minimum of $1 /\left|\delta_{f}\right|$ times:

$$
\mathrm{NMRR}_{\mathrm{av}} \geq 1 /\left|\delta_{f}\right|
$$

and practically is independent of the number $n$ of averaging indications.

So, the dependence of the effects of random noise averaging and periodic components on the number $n$ of averaged indications is different.

\section{ANALYSIS OF THE COMBINED STANDARD UNCERTAINTY OF THE CORRECTION METHODS}

Advanced correction of additive effect and time drift. The sensitivity coefficients for each indication in (3) are as follows:

$$
C_{\mathrm{N}_{\mathrm{x} 1}}=C_{\mathrm{N}_{\mathrm{x} 2}}=C_{\mathrm{N}_{\mathrm{x} 3}}=C_{\mathrm{N}_{\mathrm{x} 4}}=1 / 4
$$

In $1^{\text {st }}$ and $4^{\text {th }}$ measurements $N_{\mathrm{x} 1} \approx N_{\mathrm{x} 4}$ (measurements of $+x)$, therefore, the effects of gain errors and integral nonlinearity in these measurements are approximately the same,

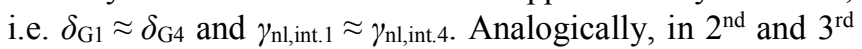
measurements $N_{\mathrm{x} 2} \approx N_{\mathrm{x} 3}$ (measurements of $-x$ ) and we may assume that $\delta_{\mathrm{G} 2} \approx \delta_{\mathrm{G} 3}$ and $\gamma_{\mathrm{nl}, \text { int, } 2} \approx \gamma_{\mathrm{nl} \text {,int.3. The same effect in }}$ both pairs of measurements is present in the switches, i.e.: $\Delta \mathrm{e}_{\mathrm{sw} 1} \approx \Delta \mathrm{e}_{\mathrm{sw} 4}$ and $\Delta \mathrm{e}_{\mathrm{sw} 2} \approx \Delta \mathrm{e}_{\mathrm{sw} 3}$.

Assuming that special processing of the registered observations is not used, i.e. $A_{\mathrm{sp}, 3}(f)=1$, using (8), (9), (10), (12), (13), (26) and also the values $A_{\text {ext,3}}(f)$ and $A_{\mathrm{int}, 3}(f)$, and coefficients $C_{3}^{2}$ in the first row in Table 3, the combined standard uncertainty caused by the correction method (3) can be presented by formula:

$$
\begin{aligned}
& u_{\mathrm{c}}^{2}(x)_{3}=\frac{\left(2^{-2(n+1)}\left(1+a_{\mathrm{nl}, \mathrm{dif}}\right)^{2}+2 \gamma_{\mathrm{nl}, \mathrm{int}}^{2}\right) X_{\mathrm{R}}^{2}+2 \delta_{\mathrm{G}}^{2} x_{\mathrm{cor}}^{2}+2 \Delta e_{\mathrm{sw}}^{2}}{12}+ \\
& +\frac{N_{0, \mathrm{ext}} \cdot B_{\mathrm{ext}}+N_{0, \mathrm{int}} \cdot B_{\mathrm{int}}}{4}+\frac{X_{\mathrm{m}, \mathrm{ext}}^{2} A_{\mathrm{ext}, 3}^{2}+X_{\mathrm{m}, \mathrm{int}}^{2} A_{\mathrm{int}, 3}^{2}}{2}
\end{aligned}
$$

where $\delta_{\mathrm{G}}$ is the maximal permissible value of gain error of the measuring chain.

Standard correction of additive and multiplicative effects. In method (4) the impact of linear time drift is not corrected. When determining the sensitivity coefficients, we assume simplifications: $N_{0}<N_{x} \approx x, N_{0}<N_{\text {ref }}, N_{\text {ref }} \approx X_{\text {ref }}$, and as previously $x \approx x_{\text {cor }}$. Therefore, the sensitivity coefficients in (4) are:

$$
C_{N_{x}} \approx 1, C_{N_{\mathrm{ref}}} \approx-\frac{x_{\mathrm{cor}}}{X_{\mathrm{ref}}}, C_{N_{0}} \approx \frac{x_{\mathrm{cor}}}{X_{\mathrm{ref}}}-1
$$

The sum of squares of these coefficients is:

$$
\sum_{i} C_{i}^{2}=2\left(1-\frac{x_{\mathrm{cor}}}{X_{\mathrm{ref}}}+\left(\frac{x_{\mathrm{cor}}}{X_{\mathrm{ref}}}\right)^{2}\right)=C_{4}^{2}=C_{4, \mathrm{int}}^{2}
$$

In this method from the point of view of the correction of additive and multiplier effects, the sequence of three measurements does not matter. However, from the point of view of the influence of drift and internal periodical interferences, the sequences of measurements are important. The minimal impact of a time drift is obtained in the first measurement sequences (1) in Table 2,a. If in second and third measurements the values of drift are $s_{\mathrm{dr}} \cdot 1 T$ and $s_{\mathrm{dr}} \cdot 2 T$, then the component of standard uncertainty is given as:

$$
u(x)_{\mathrm{dr}, 1} \approx \frac{\left|s_{\mathrm{dr}}\right| T}{\sqrt{3}}\left|1-2 \frac{x_{\mathrm{cor}}}{X_{\mathrm{ref}}}\right|
$$


In the second and third sequences of measurements (Table 2,a) the uncertainty components caused by linear time drift are given by formulas, respectively:

$$
u(x)_{\mathrm{dr}, 2} \approx \frac{\left|s_{\mathrm{dr}}\right| T}{\sqrt{3}}\left|2-\frac{x_{\mathrm{cor}}}{X_{\mathrm{ref}}}\right|, u(x)_{\mathrm{dr}, 3} \approx \frac{\left|s_{\mathrm{dr}}\right| T \mid}{\sqrt{3}}\left|1+\frac{x_{\mathrm{cor}}}{X_{\mathrm{ref}}}\right|
$$

We can see that when $0<x<X_{\text {ref }}$ in (30), the influence of time drift on the uncertainty of the corrected result is always less in comparison with (31).

Assuming $A_{s p}(f)=1$ and taking into account (28) - (30) and values in the second row in Table 3 , the combined standard uncertainty caused by the correction method (4) can be presented by formula:

$$
\begin{gathered}
u_{\mathrm{c}}^{2}(x)_{4}=\frac{1}{3}\left[\begin{array}{l}
\left(\left(2^{-2(n+1)}\left(1+a_{\mathrm{nl}, \mathrm{dif}}\right)^{2}+\gamma_{\mathrm{nl}, \mathrm{int}}^{2}\right) X_{\mathrm{R}}^{2}+\Delta e_{\mathrm{sw}}^{2}\right) \cdot C_{4}^{2}+ \\
\left(s_{\mathrm{dr}} T\right)^{2}\left(1-2 x_{\mathrm{cor}} / X_{\mathrm{ref}}\right)^{2}+\left(x_{\mathrm{cor}} \cdot \delta_{\mathrm{ref}}\right)^{2}
\end{array}\right]+ \\
+B_{\mathrm{ext}} N_{0, \mathrm{ext}}+C_{4}^{2} B_{\mathrm{int}} N_{0, \mathrm{int}}+\frac{X_{\mathrm{m}, \mathrm{xx}}^{2}+X_{\mathrm{m}, \mathrm{int}}^{2} A_{\mathrm{int}, 4}^{2}}{2} .
\end{gathered}
$$

In this case, after suppressing noises and interferences in addition to non-linearity and switches, the limitation of correction efficiency is associated with the uncertainty of reference quantity and drift effects.

Joint correction of additive and multiplicative effects and linear time drift. In correction methods (5) and (6) the impact of systematic additive and multiplicative influences and also linear time drift are theoretically corrected completely. To calculate the uncertainty, we have previously determined the values of the sensitivity coefficients. For both correction methods (5) and (6), sensitivity coefficients can be determined assuming approximations: $N_{\mathrm{x} 1} \approx-N_{\mathrm{x} 2}$, $N_{\text {ref } 1} \approx-N_{\text {ref } 2} \approx X_{\text {ref }}$, and $x \approx x_{\text {cor }}$. Then for the correction method (5) sensitivity coefficients are:

$$
\left.\begin{array}{l}
c_{N_{x 1}} \approx-\frac{x_{\mathrm{cor}}}{2 X_{\mathrm{ref}}}, c_{N_{\mathrm{x} 2}} \approx-\left(1+\frac{x_{\mathrm{cor}}}{2 X_{\mathrm{ref}}}\right), \\
c_{N_{\mathrm{ref} 1}} \approx\left(\frac{1}{2}+\frac{x_{\mathrm{cor}}}{X_{\text {ref }}}\right), \quad c_{N_{\mathrm{ref} 2}} \approx \frac{1}{2} .
\end{array}\right\} .
$$

For the correction method (6) sensitivity coefficients are given by formulae [14]:

$$
\left.\begin{array}{l}
c_{N_{\mathrm{x} 1}} \approx \frac{1}{8}\left(3-\frac{x_{\mathrm{cor}}}{X_{\mathrm{ref}}}\right), c_{N_{\mathrm{x} 2}} \approx \frac{-1}{8}\left(5+\frac{x_{\mathrm{cor}}}{X_{\mathrm{ref}}}\right), \\
c_{N_{\mathrm{ref} 1}} \approx \frac{1}{8}\left(1+\frac{5 x_{\mathrm{cor}}}{X_{\mathrm{ref}}}\right), c_{N_{\mathrm{ref} 2}} \approx \frac{1}{8}\left(1-\frac{3 x_{\mathrm{cor}}}{X_{\mathrm{ref}}}\right) .
\end{array}\right\}
$$

The sums of squares of these coefficients are:

$$
\left.\begin{array}{l}
\sum_{i} C_{5, i}^{2} \approx \frac{3}{2}\left(1+\frac{4}{3} \frac{x_{\mathrm{cor}}}{X_{\mathrm{ref}}}+\left(\frac{x_{\mathrm{cor}}}{X_{\mathrm{ref}}}\right)^{2}\right)=C_{5}^{2}=C_{5, \mathrm{int}}^{2}, \\
\sum_{i} C_{6, i}^{2} \approx \frac{9}{16}\left(1+\frac{2}{9} \frac{x_{\mathrm{cor}}}{X_{\mathrm{ref}}}+\left(\frac{x_{\mathrm{cor}}}{X_{\mathrm{ref}}}\right)^{2}\right)=C_{6}^{2}=C_{6, \mathrm{int}}^{2} \cdot
\end{array}\right\}
$$

For both correction methods (5) and (6), sensitivity coefficient $c_{\text {ref }} \approx x_{\text {cor }} / X_{\text {ref }}$. Therefore, assuming $A_{s p}(f)=1$ and using the value

$$
u_{\mathrm{q}, \mathrm{n}, \mathrm{sw}}^{2}(x)=\frac{1}{3}\left(\left(2^{-2(n+1)}\left(1+\alpha_{\mathrm{nl}, \mathrm{difl}}\right)^{2}+\gamma_{\mathrm{nl}, \mathrm{int}}^{2}\right) X_{\mathrm{R}}^{2}+\Delta e_{\mathrm{sw}}^{2}\right)
$$

of uncertainty caused by the quantization effect, nonlinearity and switches system and taking into account (33) (36), the combined standard uncertainties caused by the correction methods (5) and (6) are given by formulae:

$$
\begin{aligned}
& u_{\mathrm{c}}^{2}(x)_{5}=C_{5}^{2} u_{\mathrm{q}, \mathrm{n}, \mathrm{sw}}^{2}(x)+\frac{1}{3}\left(x_{\mathrm{cor}} \delta_{\mathrm{ref}}\right)^{2}+ \\
& +C_{5, \mathrm{ext}}^{2} B_{\mathrm{ext}} N_{0 \mathrm{ext}}+C_{5}^{2} B_{\mathrm{int}} N_{0 \mathrm{int}}+\frac{X_{\mathrm{m}, \mathrm{ext}}^{2} A_{\mathrm{ext}, 5}^{2}+X_{\mathrm{m}, \mathrm{int}}^{2} A_{\mathrm{int}, 5}^{2}}{2}, \\
& u_{\mathrm{c}}^{2}(x)_{6}=C_{6}^{2} u_{\mathrm{q}, \mathrm{n}, \mathrm{sw}}^{2}\left(x_{\mathrm{cor}}\right)+\frac{1}{3}\left(x_{\mathrm{cor}} \delta_{\mathrm{ref}}\right)^{2}+ \\
& +C_{6, \mathrm{ext}}^{2} B_{\mathrm{ext}} N_{0 \mathrm{ext}}+C_{6}^{2} B_{\mathrm{int}} N_{0 \mathrm{int}}+\frac{X_{\mathrm{m}, \mathrm{ext}}^{2} A_{\mathrm{ext}, 6}^{2}+X_{\mathrm{m}, \mathrm{int}}^{2} A_{\mathrm{int}, 6}^{2}}{2} .
\end{aligned}
$$

After comparison of (37) and (38) we can see that differences between them are in the values of coefficients $C_{5}^{2}$ and $C_{6}^{2}$, and also between $A_{\mathrm{ext}, 5}^{2}, A_{\mathrm{int}, 5}^{2}$ and $A_{\mathrm{ext}, 6}^{2}, A_{\mathrm{int}, 6}^{2}$. Assuming for example $x \approx 0.8 X_{\text {ref }}$ in (35) we have $C_{5}^{2} \approx 4.06, \quad C_{6}^{2} \approx 1.02, \quad$ and $C_{5} / C_{6}=\sqrt{4.06 / 1.02} \approx 2$. Therefore, correction method (6) provides twice less affect of quantization and non-linearity and also internal noises and periodic interferences in comparison with method (5). Similarly, at the same level of external noises, for example, at constant spectral density $N_{0}$, the ratios of variances of corrected result in both methods are:

$$
\sigma_{\mathrm{ext}, 5}^{2} / \sigma_{\mathrm{ext}, 6}^{2}=\int_{0}^{1 / T} N_{0} A_{\mathrm{ext}, 5}^{2}(f) d f / \int_{0}^{1 / T} N_{0} A_{\mathrm{ext}, 6}^{2}(f) d f \approx 1.90 .
$$

We can see that with the same level of external and internal noises in method (6) the variance of the corrected result is expected about 2 times smaller than in method (5).

\section{RESUlTS. NUMERIC EXAMPLE}

The next analysis is applied to the correction systematic effects in multifunction 16 bit Data Acquisition of family NI $6250 / 6251 / 6254 / 6259$ [18]. In [18] on the $V_{\mathrm{R}}=10 \mathrm{~V}$ range, the absolute accuracy is given as follows: Residual Gain 
Error $(\mathrm{ppm}$ of Reading $)=60$; Gain Tempco $\left(\mathrm{ppm} /{ }^{\circ} \mathrm{C}\right)=13$; Reference Tempco $\left(\mathrm{ppm} /{ }^{\circ} \mathrm{C}\right)=1$; residual Offset Error $(\mathrm{ppm}$ of Range $)=10$; Offset Tempco $\left(\mathrm{ppm}\right.$ of Range $\left./{ }^{\circ} \mathrm{C}\right)=21$;

INL Error - non-linearity $(\mathrm{ppm}$ of Range $)=60$. Absolute uncertainty at full scale on the analog input channel after two years from the device's external calibrations is determined using following assumptions: temperature change from last external calibration $\Delta \theta e=10^{\circ} \mathrm{C}$; temperature change from last internal calibration $\Delta \theta i=1{ }^{\circ} \mathrm{C}$ [18]. Using these parameters when $V_{\mathrm{x}}=10 \mathrm{~V}$ the MPE is: GainError $\cdot V_{\mathrm{R}}+$ (OffsetError $+\mathrm{INL}$ Error) $\cdot V_{\mathrm{R}}=1840 \mu \mathrm{V}$.

Type $\mathrm{B}$ standard uncertainty (assuming uniform distribution): $1840 / \sqrt{3} \approx 1062 \mu \mathrm{V}$ (Table 4).

Random Noise $\sigma_{\mathrm{n}}(\mu \mathrm{Vrms})$ for a number of readings $n=1 ; 25 ; 100$ is [18]: $280 / \sqrt{n} \mu \operatorname{Vrms}$ (Table 4 ).

Table. Simplified budget of the combined standard uncertainty for NI $6250 / 6251 / 6254 / 6259\left(V_{\mathrm{x}} \approx V_{\mathrm{R}} \approx V_{\text {ref }}=10.0 \mathrm{~V}\right)$.

\begin{tabular}{|c|c|c|c|c|c|c|}
\hline $\begin{array}{c}\text { Standard } \\
\text { uncertainty } \\
\text { component } \\
u\left(x_{i}\right)\end{array}$ & Source of uncertainty & Type & $\begin{array}{l}\text { Distri- } \\
\text { bution }\end{array}$ & $\begin{array}{l}\text { Value of standard } \\
\text { uncertainty of } \\
\text { source: } u\left(x_{i}\right)\end{array}$ & $\begin{array}{l}\text { Sensitivity } \\
\text { coefficients } \\
c_{i}=\frac{\partial f}{\partial x_{i}} \\
\end{array}$ & $\begin{array}{c}\text { Value of } \\
\text { standard } \\
\text { uncertainty: } \\
u_{i}\left(V_{x}\right)=\left|c_{i}\right| u\left(x_{i}\right)\end{array}$ \\
\hline \multicolumn{7}{|c|}{ When correction is not used, after data in [18] } \\
\hline$u\left(\Delta_{O S}\right)$ & Offset + Gain errors & $\mathrm{B}$ & uniform & $\begin{array}{c}\frac{101 \cdot 10+83 \cdot 10}{\sqrt{3}} \approx 1 \\
062 \mu \mathrm{V}\end{array}$ & 1 & $\approx 1062 \mu \mathrm{V}$ \\
\hline$u(\eta)$ & $\begin{array}{l}\text { Nosie: } 280 \mu \mathrm{Vrms} \\
n=1 \\
n=25 \\
n=100\end{array}$ & A & normal & $\begin{array}{c}(37) \\
n=1: 280 \mu \mathrm{V} \\
n=25: 56 \mu \mathrm{V} \\
n=100: 28 \mu \mathrm{V}\end{array}$ & 1 & $\begin{array}{l}280 \mu \mathrm{V} \\
56 \mu \mathrm{V} \\
28 \mu \mathrm{V}\end{array}$ \\
\hline$u(\zeta)$ & $\begin{array}{l}\text { Normal and common } \\
\text { interference of } 50 \mathrm{~Hz}, \\
V_{\mathrm{nm}}=1 \mathrm{mVrms}, \mathrm{NMRR}=100 \\
\left(\left|\delta_{\mathrm{f}}\right|=0.01, n=25,100\right), \\
V_{\mathrm{cm}}=10 \mathrm{Vrms}, \mathrm{CMMR}=10^{5} .\end{array}$ & A & arcsine & $\begin{array}{c}\quad(39) \\
n=1: 280 \mu \mathrm{V} \\
n=25: 11 \mu \mathrm{V} \\
n=100: 11 \mu \mathrm{V}\end{array}$ & 1 & $\begin{array}{c}1100 \mu \mathrm{V} \\
11 \mu \mathrm{V} \\
11 \mu \mathrm{V}\end{array}$ \\
\hline$u_{c}\left(V_{\mathrm{x}}\right)$ & $\begin{array}{l}\text { Combined uncertainty of } \\
\text { uncorrected result }\end{array}$ & $\mathrm{A}+\mathrm{B}$ & \multicolumn{4}{|c|}{$\begin{array}{c}n=1: 1555 \mu \mathrm{V} \\
n=25: 1064 \mu \mathrm{V} \\
n=100: 1063 \mu \mathrm{V} \\
\end{array}$} \\
\hline \multicolumn{7}{|c|}{ When correction is used } \\
\hline$u\left(V_{r e f}\right)$ & $\begin{array}{l}\text { Uncertainty of reference } \\
\text { voltage } V_{\text {ref }}=10 \mathrm{~V} \text { : } \\
50 \text { ppm of } V_{\text {ref }}\end{array}$ & $\mathrm{B}$ & uniform & $\begin{array}{l}\frac{50 \cdot 10 \mathrm{~V}}{\sqrt{3}} \approx \\
\approx 289 \mu \mathrm{V}\end{array}$ & 1 & $\approx 289 \mu \mathrm{V}$ \\
\hline$u(q)$ & Quantization & $\mathrm{B}$ & uniform & $\begin{array}{l}\frac{10}{2^{16} \sqrt{12}} \approx \\
\approx 44 \mu \mathrm{V}\end{array}$ & 1 & $\approx 44 \mu \mathrm{V}$ \\
\hline$u(V)_{\mathrm{nl} \text {.int }}$ & Integral nonlinearity & $\mathrm{B}$ & uniform & $\begin{array}{l}60 \cdot 10 / \sqrt{3} \approx \\
\approx 346 \mu \mathrm{V}\end{array}$ & 1 & $\approx 346 \mu \mathrm{V}$ \\
\hline$u(\eta)$ & $\begin{array}{l}\text { Nosie: } 280 \mu \mathrm{Vrms} \\
n=1 \\
n=25 \\
n=100\end{array}$ & A & normal & $\begin{array}{c}n=1: 280 \mu \mathrm{V} \\
n=25: 56 \mu \mathrm{V} \\
n=100: 28 \mu \mathrm{V}\end{array}$ & 1 & $\begin{array}{l}280 \mu \mathrm{V} \\
56 \mu \mathrm{V} \\
28 \mu \mathrm{V}\end{array}$ \\
\hline$u(\zeta)$ & $\begin{array}{l}\text { Normal and common } \\
\text { interference of } 50 \mathrm{~Hz}, \\
V_{\mathrm{m}}=1 \mathrm{mVrms}, \mathrm{NMRR}=100 \\
\left(\left|\delta_{\mathrm{f}}\right|=0.01, n=25,100\right) \\
V_{\mathrm{cm}}=10 \mathrm{Vrms}, \mathrm{CMMR}=10^{5}\end{array}$ & A & arcsine & $\begin{array}{c}n=1: 1100 \mu \mathrm{V} \\
n=25: 11 \mu \mathrm{V} \\
n=100: 11 \mu \mathrm{V}\end{array}$ & 1 & $\begin{array}{c}1100 \mu \mathrm{V} \\
11 \mu \mathrm{V} \\
11 \mu \mathrm{V}\end{array}$ \\
\hline$u_{c}\left(V_{\mathrm{x}}\right)_{\mathrm{cor}}$ & Combined standard uncertainty & $A+B$ & \multicolumn{4}{|c|}{$\begin{array}{c}n=1: 1222 \mu \mathrm{V} \\
n=25: 457 \mu \mathrm{V} \\
n=100: 454 \mu \mathrm{V}\end{array}$} \\
\hline
\end{tabular}


Normal mode interference $50 \mathrm{~Hz}$ without averaging is $1000 \mu \mathrm{Vrms}$. Using averaging of $n=25$ or 100 readings at time averaging $T_{\mathrm{av}}=20 \mathrm{~ms}$, and assuming maximal frequency deviation $\left|\delta_{f}\right|=1 \%$ from $50 \mathrm{~Hz}$, the value $\mathrm{NMRR}=1 /\left|\delta_{f}\right|=100$. After averaging $V_{\mathrm{nm}}=1 \mathrm{mVrms} / 100=$ $10 \mu$ Vrms.

Common mode voltage $V_{\mathrm{cm}}=10 \mathrm{Vrms}, \mathrm{CMRR}=100 \mathrm{~dB}$ $(50 \mathrm{~Hz})$, without averaging $10 / 10^{5}=100 \mu \mathrm{Vrms}$ and after averaging (CMRR+NMRR): $10 /\left(10^{5} \cdot 10^{2}\right)=1 \mu$ Vrms. The sum is $11 \mu$ Vrms (Table 4).

The expected values of the components of combined standard uncertainty of measurement when correction is not used are presented in the first part of Table 4. The analogical components of combined standard uncertainty of the measurement with correction by method (4) are presented in the second part of Table 4 .

The effectiveness of the correction of systematic components when number of averaged readings $n=1$, $n=25$, and $n=100$, averaged readings $\left(T_{\mathrm{av}}=20 \mathrm{~ms}\right)$ are:

$$
\begin{gathered}
E_{\mathrm{cor}, n=1}=\frac{1555 \mu \mathrm{V}}{1222 \mu \mathrm{V}} \approx 1.27 ; E_{\mathrm{cor}, n=25}=\frac{1064 \mu \mathrm{V}}{457 \mu \mathrm{V}} \approx 2.33, \\
E_{\mathrm{cor}, n=100}=\frac{1063 \mu \mathrm{V}}{454 \mu \mathrm{V}} \approx 2.34 \text { times. }
\end{gathered}
$$

We can see that suppression of noises and periodical interferences during correction is very important. In order to obtain effectiveness of the correction, the external, internal noises and periodic interferences should be suppressed by averaging of 25 readings as minimum (or by filtering). When $n=100$ readings are averaged then the efficiency of correction practically is the same as for the $n=25$ averaged readings, because at $n=100$ the $50 \mathrm{~Hz}$ interference is suppressed in the same way as at $n=25$.

However, the non-linearity of ADC and uncertainty of the value of reference voltage essentially limited the efficiency of correction. Uncertainty components caused by nonlinearity and uncertainty of reference quantity are: $u_{\mathrm{c}}\left(V_{x}\right)_{\text {cor,nl }} \approx 346 \mu \mathrm{V}, \quad u_{\mathrm{c}}\left(V_{x}\right)_{\text {cor, ref }} \approx 289 \mu \mathrm{V}$. If the nonlinearity and uncertainty of reference voltage is two times less $(30 \mathrm{ppm}$ and $25 \mathrm{ppm})$, then effectiveness of the correction at $n=100$ averaged readings would be:

$$
E_{\mathrm{cor}, n=100}=\frac{1063 \mu \mathrm{V}}{232 \mu \mathrm{V}} \approx 4.59 \text { times. }
$$

In practice of industrial measurement of nonelectrical quantities besides the Data Acquisition module, the appropriate signal conditioning modules are used, therefore the non-linearity and noises of such modules should be taken into account in the analysis of the efficiency of correction. Because in such measurements the level of input signal is in $\mathrm{mV}$ range, then the influence of switches contact voltage of a few microvolts must be taken into account in the uncertainty analysis.

\section{DISCUSSION / CONCLUSIONS}

The potential effectiveness of the correction is always limited by uncertainty of reference quantity. The main factors that limit the effectiveness of automatic correction of systematic effects and time drifts are: nonlinearity and quantization of ADC, non-idealities of switches systems, and external and internal noises and periodical interferences.

The impact of noises and periodic interferences on the combined standard uncertainty depends on the amplitude responses that are different for external and internal noises and periodical interferences, and are dependent on the algorithm of correction.

Due to different values of sensitivity coefficients and also due to different affects of internal and external noises and periodical interferences, the uncertainty of measurement with correction depends on sequences of measurement carried out to obtain the corrected result.

Since the influence of noise and interference can be reduced by means of filtration and averaging, the main factors (beside uncertainty of reference quantity) limiting the effectiveness of systematic interaction correction are the non-linearity of the function and the non-ideal parameters of the switches.

The results of a numeric example of uncertainty analyses of correction by joint correction of additive and multiplicative systematic effects applied to the multifunction 16 bit Data Acquisition of family NI 6250/6251/6254/6259 confirmed the results of previous researches. The effectiveness of the correction of the offset and gain errors is only about 2.3 times, because integral non-linearity is quite large and uncertainty of reference voltage is not sufficient.

\section{Acknowledgements:}

This work was supported by Rzeszów University of Technology under project DS.EM.18.001 and by Polish Ministry of Science and Higher Education under the program "Regional Initiative of Excellence” in 2019-2022, project number 027/RID/2018/19, funding amount 11999 900 PLN.

\section{REFERENCES}

[1] Joint Committee for Guides in Metrology. (2008). Evaluation of measurement data - Guide to the Expression of Uncertainty in Measurement, 1st edition. JCGM 100:2008.

[2] Pavese, F. (2012). On the difference of meaning of 'zero correction': zero value versus no correction, and of the associated uncertainties. In Advanced Mathematical and Computational Tools in Metrology IX. World Scientific, 297-309.

[3] Pavese, F. (2013). Why should correction values be better known than the measurand true value? Journal of Physics: Conference Series, 459, 012036. 
[4] Pavese, F. (2012). Corrections and input quantities in measurement models. International Journal of Metrology and Quality Engineering, 3, 155-159.

[5] Danilov, A.A., Kurchenko, Yu. (2015). Calibration method of measuring instruments in operating conditions. In Advanced Mathematical and Computational Tools in Metrology X. World Scientific, 149-155.

[6] Krilov, A., Marinko, S, Boyko, L. (2015). Considerations on the influence of test equipment instability and calibration methods on measurement uncertainty of the test laboratory. In Advanced Mathematical and Computational Tools in Metrology $X$. World Scientific, 219-228.

[7] Pavese, F. (2018). On the classification into random and systematic effects. In Advanced Mathematical and Computational Tools in Metrology and Testing XI. World Scientific, 58-69.

[8] Keithley Instruments. Low Level Measurements Handbook - $7^{\text {th }}$ Edition : Precision DC Current, Voltage and Resistance Measurements.

[9] Meijer, G.C.M. (ed.) (2008). Smart Sensor Systems. John Wiley and Sons.

[10] Tuz, Ju.M. (1976). Structural Methods to Improve the Accuracy of Measuring Devices. Kyiv: Vyshcha shkola. (in Rusian)

[11] Bromberg, E.M. (1978). Test Methods for Improving the Accuracy of Measurements. Moscow: Energia. (in Russian)
[12] Wang, G., Meijer, G.C. (2000). Accurate DEM SC amplification of small differential-voltage with CM level from ground to VDD. In Smart Structures and Materials 2000: Smart Electronics and MEMS. SPIE, Vol. 3990. DOI: 10.1117/12.388913.

[13] Keithley Instruments. (2008). Switching Handbook: A Guide to Signal Switching in Automated Test Systems, $6^{\text {th }}$ Edition.

[14] Dorozhovets, M. (2015). Uncertainty of correction of the systematic effects and drifts. Przeglad Elektrotechniczny, 11, 300-304. (in Polish)

[15] Bayley, G.V., Hammersley, G.M. (1946). The "effective" number of independent observations in an autocorrelated time-series. Supplement to the Journal of the Royal Statistical Society, 8 (2), 184-197.

[16] Zhang, N.F. (2006). Calculation of the uncertainty of the mean of autocorrelated measurements. Metrologia 43 (4), 276-281.

[17] Warsza, Z., Dorozhovets, M. (2008). Uncertainty type A evaluation of autocorrelated measurement observations. Biuletyn WAT, LVII (2), 143-152.

[18] National Instruments Corporation. NI 6250/6251/6254/6259 Multifunction 16 bit Data Acquisition. Datasheet. http://www.ni.com.

Received March 20, 2019 Accepted July 31, 2019 